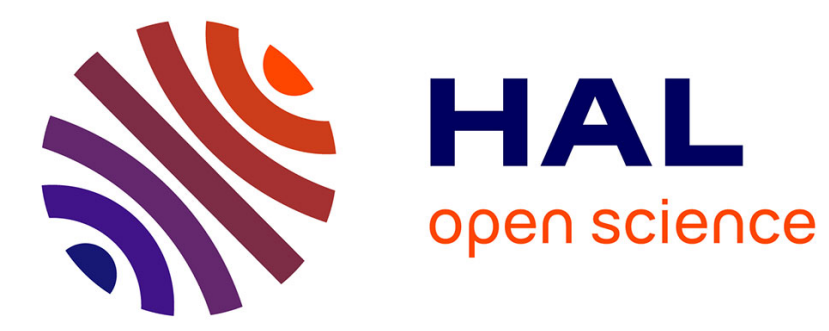

\title{
Effect of upstream turbulence on single and dual- stream jets. Assessment of Zonal Detached Eddy Simulation (ZDES)
}

Fabien Gand, J. Verrière, Sébastien Deck

\section{> To cite this version:}

Fabien Gand, J. Verrière, Sébastien Deck. Effect of upstream turbulence on single and dual- stream jets. Assessment of Zonal Detached Eddy Simulation (ZDES). 6th Symposium on Hybrid RANS-LES methods, Sep 2016, STRASBOURG, France. hal-01411391

\author{
HAL Id: hal-01411391 \\ https://hal.science/hal-01411391
}

Submitted on 7 Dec 2016

HAL is a multi-disciplinary open access archive for the deposit and dissemination of scientific research documents, whether they are published or not. The documents may come from teaching and research institutions in France or abroad, or from public or private research centers.
L'archive ouverte pluridisciplinaire $\mathbf{H A L}$, est destinée au dépôt et à la diffusion de documents scientifiques de niveau recherche, publiés ou non, émanant des établissements d'enseignement et de recherche français ou étrangers, des laboratoires publics ou privés. 


\title{
Effect of upstream turbulence on single and dual- stream jets. Assessment of Zonal Detached Eddy Simulation (ZDES).
}

\author{
F. Gand ${ }^{1}$, J. Verrière ${ }^{1,2}$ and S. Deck ${ }^{1}$
}

Abstract This paper aims at investigating the effect of upstream turbulence (for instance coming from the engine's internal geometry) on the jet flow development. Simulations are performed using ZDES [1] combined with synthetic turbulence generation methods. Two cases are studied: an incompressible, singlestream jet and a compressible dual-stream one. It is shown that the upstream turbulence reduces the RANS-to-LES transition and improves the prediction of the location of the shock-cells and global jet flow development, which advocates a systematic consideration of realistic nozzle exit conditions in eddy-resolving simulations.

\section{Introduction}

Hybrid simulations, and more generally eddy-resolving ones, are now widely used in the aeronautic industry [2]. The commonly used RANS/LES approaches (switching from RANS in attached areas to LES in detached ones) rely on the natural flow instabilities to trigger the development of the resolved turbulence in shear layers. These instabilities are rather weak in the case of jet flows, which leads to large delays in the RANS-to-LES transition and poor aerodynamic and aeroacoustic predictions. Some improvements of DES formulation have been proposed to mitigate this delay [1] [3].

Another issue related to the use of eddy-resolving simulations for jet flows is the consideration of freestream turbulence in the jets core, which is inevitable in experiments and comes from the upstream parts of the engine in flight configurations. Few published studies have tackled this issue with different methods [4] [5] [6]. It is noteworthy that, in all these cases, the introduction of upstream turbulence also improved the RANS-to-LES transition.

Therefore, the objective of this paper is to investigate the effect of adding freestream turbulence targeted at reproducing realistic inflow conditions on the turbulence development in mixing layers within the framework of ZDES simulations of jets. This is assessed on two configurations: a single stream, low speed jet and a more realistic dual-stream nozzle at high Reynolds number.

${ }^{1}$ ONERA - The French Aerospace Lab, F-92190 Meudon, France

${ }^{2}$ Airbus Operations SAS, Toulouse, France 


\section{Zonal Detached Eddy Simulation (ZDES)}

In this paper, hybrid RANS/LES simulations are performed using the Zonal Detached Eddy Simulation (ZDES) [1]. Modes 1 and 2 of the ZDES are used. The ZDES mode 1, introduced to treat separations triggered by the geometry, relies on a user-defined zonal decomposition of the computational domain in RANS and DES areas. The hybrid length scale $\tilde{d}_{Z D E S}^{I}$ used for the Spalart-Allmaras (SA) model is equal to:

$$
\tilde{d}_{\text {ZDES }}^{I}=\left\{\begin{array}{l}
d_{\text {wall }} \text { in RANS areas } \\
\min \left(d_{\text {wall }}, C_{D E S} \tilde{\Delta}_{\text {ZDES }}^{I}\right) \text { in DES areas }
\end{array}\right.
$$

where $d_{\text {wall }}$ is the wall distance; $C_{D E S}$ is the original DES97 constant [7] and $\tilde{\Delta}_{\text {ZDES }}^{I}=\Delta_{\text {vol }}=(\Delta \mathrm{x} \Delta \mathrm{y} \Delta \mathrm{z})^{1 / 3}$ or $\tilde{\Delta}_{\text {ZDES }}^{I}=\Delta_{\omega}=\sqrt{\bar{S}_{\omega}}, \bar{S}_{\omega}$ is the average cross section of the cell normal to the vorticity vector $\omega$. In the present study, the subgrid length scale based on the local vorticity vector direction $\Delta_{\omega}$ was chosen since there is a preferred mean vorticity direction. Furthermore, the near-wall functions of the SA model are removed in LES areas for mode 1: $f_{v 1}=1, f_{v 2}=0, f_{w}=0$.

The ZDES mode 2 was developed to deal with separations over smooth surfaces and works as a global approach where the model itself defines the RANS and DES areas, which makes the treatment of complex geometries straightforward for the user. To ensure that attached boundary layers are treated in RANS whatever the grid density, a shielding function $f_{d}$ is used - similar to the one employed in DDES [8] - to shield the attached boundary layers. The hybrid length scale reads:

$$
\tilde{d}_{\text {ZDES }}^{I I}=d_{\text {wall }}-f_{d} \max \left(0, d_{\text {wall }}-C_{D E S} \tilde{\sim}_{\text {ZDES }}^{I I}\right)
$$

Conversely to DDES, the mode 2 of ZDES provides a specific definition of the subgrid length scale according to the flow resolution and a threshold value $f_{d}=0.8$ determined in Ref [1]. When $\mathrm{f}_{\mathrm{d}}<\mathrm{f}_{\mathrm{d} 0}, \tilde{\Delta}_{\text {ZDES }}^{I I}=\Delta_{\max }\left(\Delta_{\max }\right.$ being the characteristic mesh length necessary to ensure the correct behavior of $f_{d}$ ). When $\mathrm{f}_{\mathrm{d}}>\mathrm{f}_{\mathrm{d} 0}$, the subgrid length scale revolves to $\tilde{\Delta}_{Z D E S}^{I I}=\Delta_{\omega}$ or $\Delta_{v o l}$. As explained in Ref [1], the nearwall functions of the SA model are not modified in mode 2 of ZDES. 


\section{Single stream jet}

\subsection{Description of the test case and simulations}

The round jet investigated is similar to one investigated experimentally in Ref. [9]. The Reynolds number based on the nozzle exit velocity $U_{0}=20 \mathrm{~m} / \mathrm{s}$ and diameter $\mathrm{D}=0.15 \mathrm{~m}$ is equal to $\mathrm{Re}_{\mathrm{D}}=2.110^{5}$. During the experiments, the boundary layer inside the nozzle was tripped using sand paper so that the exit boundary layer is fully turbulent, of thickness $\delta_{0}=3 \mathrm{~mm}=0.02 \mathrm{D}$ and momentum thickness $\theta_{0}=0.36 \mathrm{~mm}=0.0024 \mathrm{D}$.

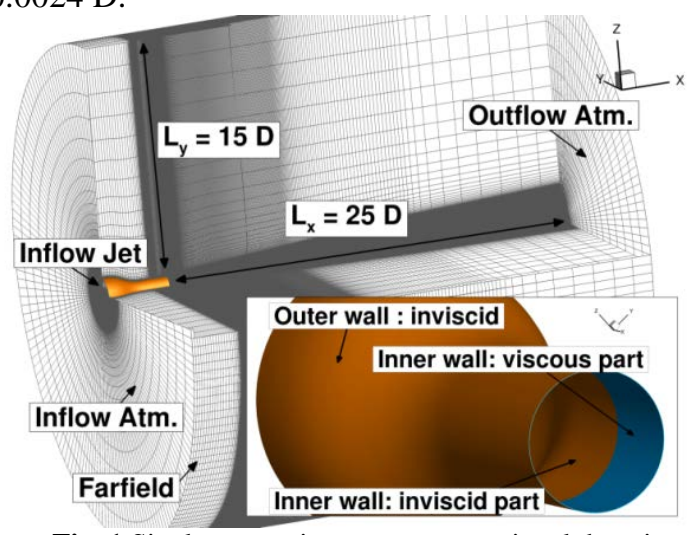

Fig. 1 Single stream jet case, computational domain

The simulations were performed with the elsA software developed at ONERA [10] which solves the compressible Navier-Stokes equations on structured multiblock meshes. A sketch of the computational domain and boundary conditions is presented in Fig. 1. 40 grid points are used to discretize the nozzle boundary layer, 360 points are used in the azimuthal direction and the mesh contains $40.10^{6}$ cells in total.

The time integration is performed using an implicit LU-SSOR algorithm and a second-order accurate backward Gear scheme with 5 Newton sub-iterations to ensure a drop of one order of magnitude of the inner-iteration residuals.

The diffusive fluxes are discretized using a second-order-accurate centered scheme. The convective terms are treated with the hybrid centered/upwind second-order-accurate AUSM+P scheme [11] using MUSCL extrapolation of the third order.

To generate a realistic turbulent inflow content, the Synthetic Eddy Method (SEM) [12] is used. The SEM is based on the generation of velocity fluctuations carried by synthetic eddies which are superimposed to a target mean flow to obtain a synthetic unsteady turbulent field used to feed an inlet boundary condition. 
The formulation relies on the prescription by the user of a target Reynolds stress tensor, which is related here to the jet core turbulence rate Tu: $\overline{u_{\imath} u_{\jmath}}=T u^{2} \times U_{0}^{2} \times$ $\delta_{i j}$ (where $\mathrm{U}_{0}$ is the inlet mean velocity and $\delta_{\mathrm{ij}}$ is the Kronecker delta). The values tested for the turbulence length scale $\sigma$ are given in Table 1 .

For all simulations, the time step is $210^{-6} \mathrm{~s}$. The unsteady results are time averaged over $200 \mathrm{~ms}(\sim 25 \mathrm{D} / \mathrm{U})$ after a transient stage estimated of around $120 \mathrm{~ms}$ ( 16 D/U).

\begin{tabular}{ccc}
\hline \hline Case & RANS/LES approach & Inflow condition \\
\hline \hline ZDES2 & ZDES (all domains : mode $2 \Delta_{\omega}$ ) & Steady uniform Pt, Tt \\
ZDES2-SEM & ZDES (all domains : mode $2 \Delta_{\omega}$ ) & SEM Jarrin $\sigma=0,02 \mathrm{Tu}=7,5 \%$ \\
ZDES2-SEM2 & ZDES (all domains : mode $2 \Delta_{\omega}$ ) & SEM Jarrin $\sigma=0,02 \mathrm{Tu}=35 \%$ \\
ZDES2-SEM3 & ZDES (all domains : mode $2 \Delta_{\omega}$ ) & SEM Jarrin $\sigma=0,05 \mathrm{Tu}=7,5 \%$ \\
\hline \hline
\end{tabular}

\subsection{Results}

In this paper, the focus is put on the major modifications in the jet development induced by the introduction of upstream turbulence. A more complete analysis and validation of these results as well as the comparison between mode 1 and 2 of ZDES and DDES is presented in Ref [5].

The shape and size of the injected eddies in the nozzle inlet plane of simulation ZDES2-SEM can be educed from Fig. 2 (b), to be compared with the uniform inlet in simulation ZDES2. The insets in Fig. 2 depict the turbulence development in the early shear layer. The spatial wavelength and transition length seem to be modified by the upstream turbulence.
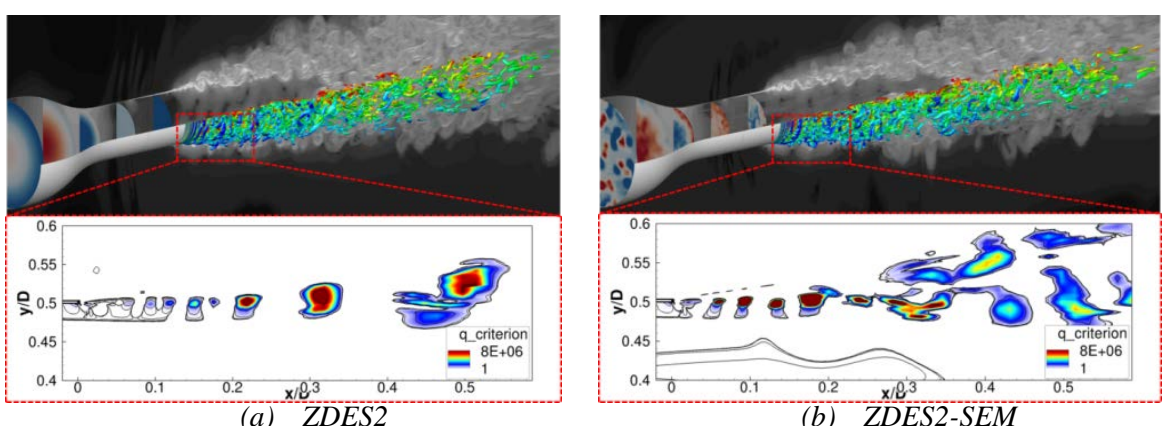

Fig. 2 Illustration of the simulations and close-up on the mixing layer early development

More quantitatively, the RMS profiles along the lip line in Fig. 3 show that the dual-peaked shape characteristic of vortex pairing is attenuated. This is attributed 
to a reduction of the RANS-to-LES transition length when upstream turbulence is introduced in the jet core, due to the penetration of resolved fluctuations into the nozzle boundary layer treated in RANS which promotes the development of LES content in the early stage of the mixing layer development. The attenuation of the vortex pairing is further evidenced by the velocity spectra in Fig. 4 near the nozzle exit, since the hump near $\mathrm{St}_{\theta}=0.013$ associated with the vortex pairing is smoothed in cases with upstream turbulence.

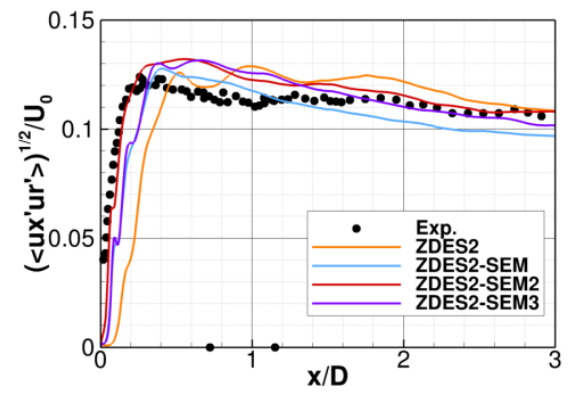

Fig. 3 Evolution of shear stresses along the lip line

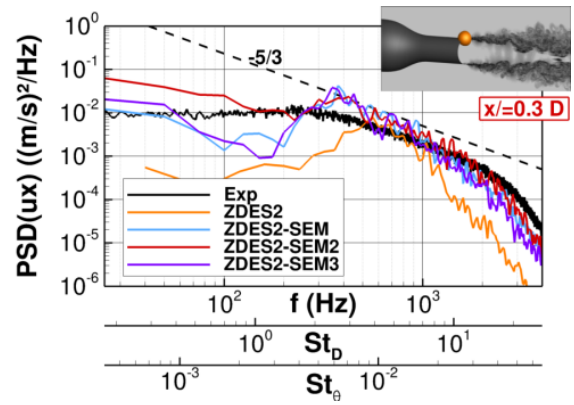

Fig. 4 Velocity spectra close to the nozzle exit

The effect of upstream turbulence on the jet flow development is presented in Fig. 5. The turbulence rate at the nozzle exit in simulation ZDES2-SEM is low but comparable to the one in the experiments, and this seems to improve the potential core length prediction compared to the simulation ZDES2 with zero turbulence rate. The increase of the turbulence rate in the simulations results in a shorter jet (ZDES2-SEM2). When larger synthetic eddies are injected (ZDES2-SEM3 compared to ZDES2-SEM with the same inlet turbulence rate), they are less dissipated by the grid in the nozzle, thus a higher turbulence rate is achieved at the nozzle exit and the jet is slightly shorter. Velocity spectra along the jet centerline in Fig. 6 show the progressive attenuation of the jet column mode at $\mathrm{St}_{\mathrm{D}}=0.6$ when the turbulence rate is increased.

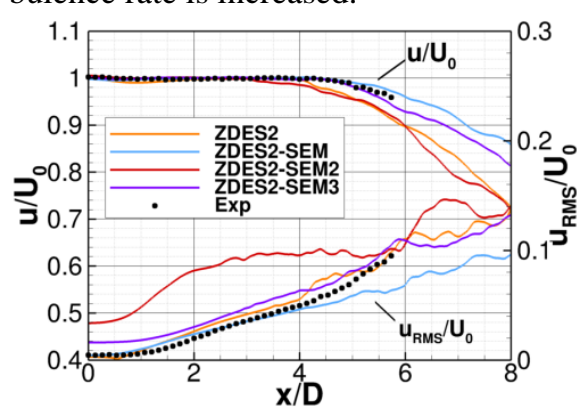

Fig. 5 Evolution of the mean velocity and RMS of velocity along the jet centerline

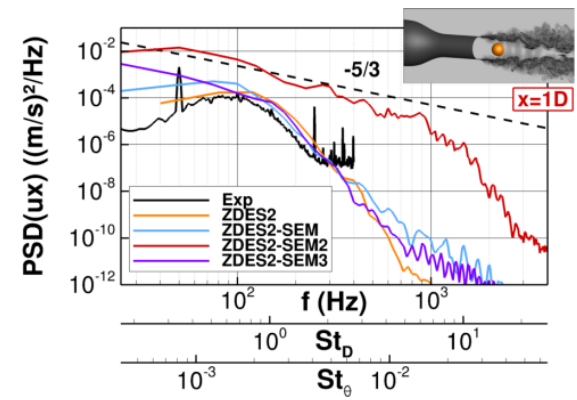

Fig. 6 Velocity spectra along the jet centerline 


\section{Dual-stream jet}

\subsection{Description of the test case and simulations}

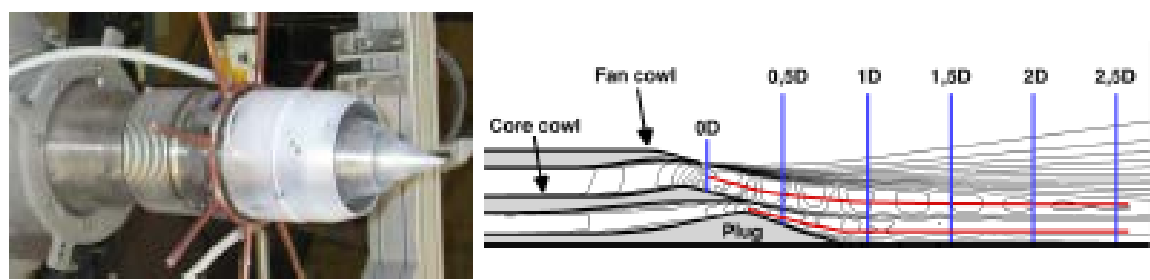

Fig. 7 Martel test case and measurement lines

The dual-stream jet considered in this paper is the MARTEL configuration which was investigated experimentally at the Pprime institute in Poitiers, France. This configuration is illustrated in Fig. 7 and consists in an isolated heated axisymmetric coaxial jet, without external flow. The details of the operating point computed are given in Ref [13].

The simulations were carried out with the elsA software [10] on a structured multi-block grid of around 205 million grid points (illustrated in Fig. 8), with 416 points in the azimuthal direction. In the mixing layers, the radial spacing is about $\Delta \mathrm{r}=\delta \omega / 40$, and the axial and azimuthal spacings verify $\Delta \mathrm{x}, \mathrm{z}<\delta \omega / 2$, where $\delta \omega$ is the shear layers vorticity thickness extracted from a preliminary RANS computation.

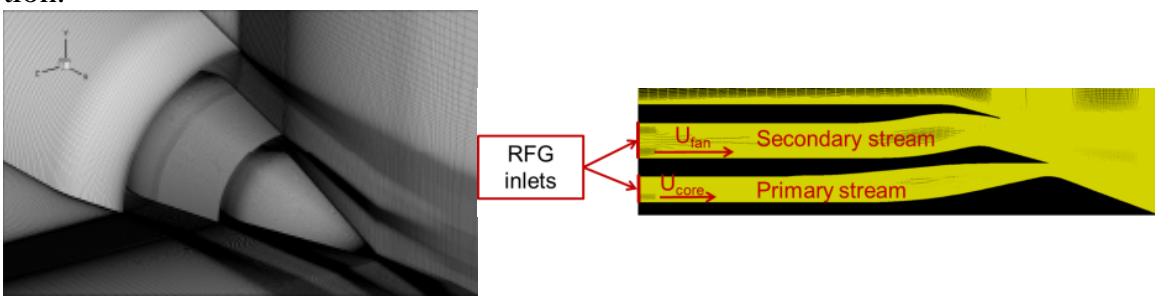

Fig. 8 ZDES grid and inlet boundary conditions for the Martel test case

The time integration is identical to the one used in the previous section (see section 3.1). The convective fluxes are treated with the centered second order scheme of Jameson with second and fourth order dissipation coefficients equal to $\chi 2=0.5$ and $\chi 4=0.008$.

In order to introduce upstream turbulence, the Random Flow Generation (RFG) technique developed by Smirnov et al. [14] is used at the nozzle internal ducts inflow boundary conditions (see Fig. 8). This method is based on the superposition of harmonic functions and has already been used in elsA simulations in previous work [4] [15]. The parameters investigated in this paper are given in Table 2, the same parameters are used for both primary and secondary duct inlets. The target 
Reynolds stress tensor is related to the turbulence rate by $\overline{u_{\imath} u_{J}}=T u^{2} \times U_{0}^{2} \times \delta_{i j}$, where $\mathrm{U}_{0}$ is the mean velocity at the inlet of the duct considered.

The physical time step used for unsteady simulations is $\Delta \mathrm{t}=1 \cdot 5 \cdot 10^{-7} \mathrm{~s}$. The spectral analysis has been carried out using a time signal of $\mathrm{T}=36 \mathrm{~ms}=116 \mathrm{D} / \mathrm{U}$, where $\mathrm{D}$ is the bypass exhaust diameter and $\mathrm{U}$ is the bypass exhaust velocity.

\begin{tabular}{ccccc}
\hline \hline Simulation & $\mathbf{J} \boldsymbol{\omega}$ & $\mathbf{J} \boldsymbol{\omega}-\mathbf{T 1 0} \mathbf{H} / \mathbf{1 0}$ & $\mathbf{J} \boldsymbol{\omega}-\mathbf{T} 5-\mathbf{H} / \mathbf{1 0}$ & $\mathbf{J} \boldsymbol{\omega}-\mathbf{T} 5-\mathbf{H} / \mathbf{5}$ \\
\hline \hline Turbulence rate & - & $10 \%$ & $5 \%$ & $5 \%$ \\
Turbulent length scale & - & $\mathrm{H} / 10$ & $\mathrm{H} / 10$ & $\mathrm{H} / 5$ \\
\hline \hline
\end{tabular}

Table 2 Simulations performed for the Martel test case. $\mathrm{H}$ is the height of the duct considered

\subsection{Results}

Fig. 9 illustrates the salient features of the baseline J $\omega$ simulation. The reader is referred to Ref [13] for a detailed validation and analysis of this simulation. The secondary mixing layer is quickly destabilized, whereas the primary one seems to suffer from a delay in the RANS-to-LES transition which may be due to the low velocity difference between the primary and secondary streams [13]. In the secondary stream, a shock-cell patterns is observed.

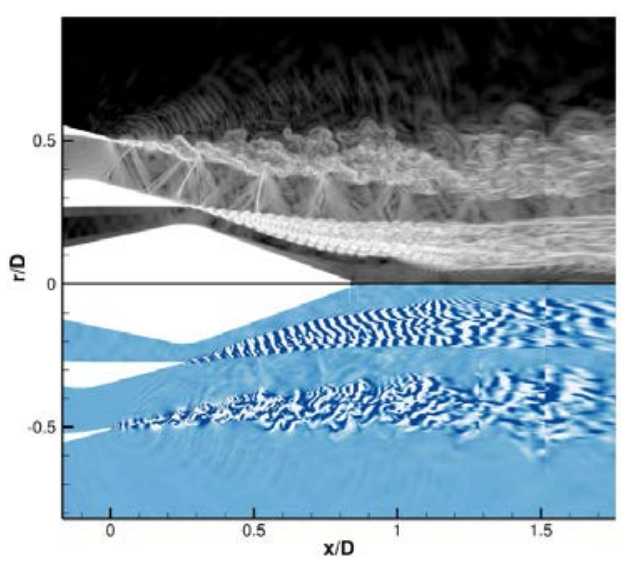

Fig. 9 Main features of the Jw simulation

The evolution of the injected turbulence in the nozzle ducts is depicted in Fig. 10. The intensity and size of the velocity field distortions are correlated with the RFG parameters and no major damping of the fluctuations is observed as they are transported within the ducts. 


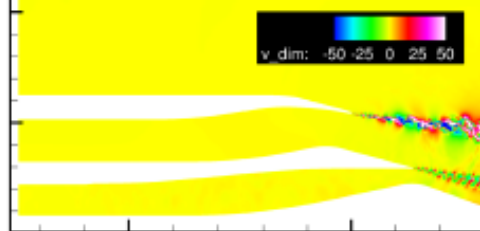

(a) $J w$

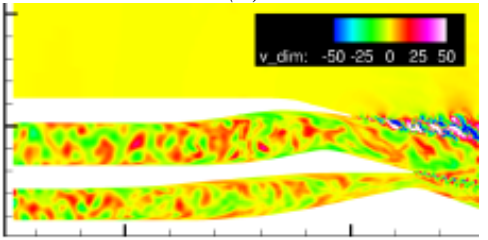

(c) $\mathrm{Jw}-\mathrm{T5}-\mathrm{H} / 10$

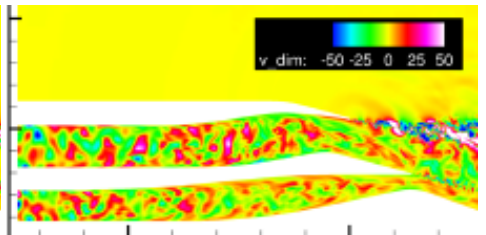

(b) $\mathrm{Jw}-\mathrm{T10}-\mathrm{H} / 10$

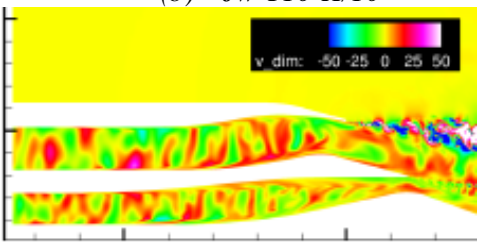

(d) $\mathrm{Jw}-\mathrm{T5}-\mathrm{H} / 5$

Fig. 10 Radial velocity fields

The RANS-to-LES transition in the secondary mixing layer can be assessed in Fig. 11. As observed in the results for the single-stream jet, secondary peaks in the profiles of turbulent kinetic energy are somewhat smoothed when upstream turbulence is introduced - even if the main peak is stronger, which suggests a more intense transition. The quicker RANS-to-LES transition leads to a better agreement between the simulations and the experiment regarding the mixing layer growth rate.

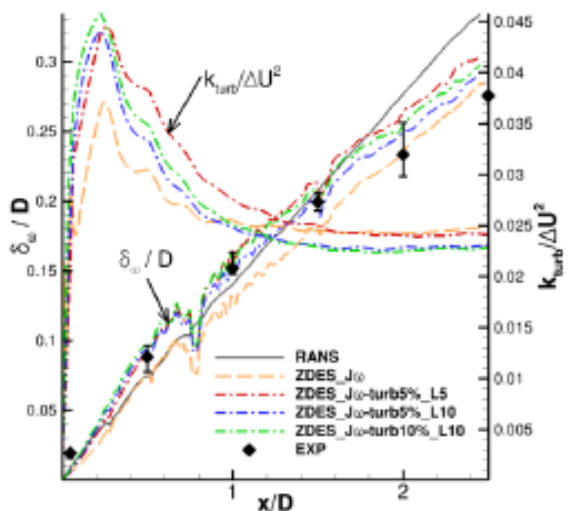

Fig. 11 Fan mixing layer streamwise evolution

The azimuthal distributions of vorticity in the jet cross plane at $\mathrm{x} / \mathrm{D}=1$ presented in Fig. 12 indicate the level of resolved turbulence outside the mixing layers in both streams. This results in increased mixing in the streams core, which in turns modifies the shock-cell positioning as shown in Fig. 13 and reduces the phase-lag between simulations and experiments. This is a major improvement in the simulations and shows the necessity of taking into account upstream turbulence for a quantitative jets aerodynamic performances prediction. 


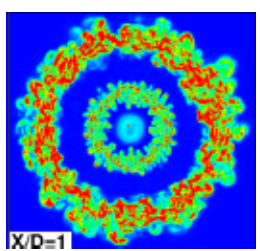

(a) $J w$

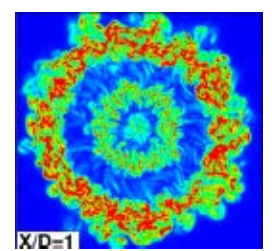

(b) $J w-T 5-H / 10$

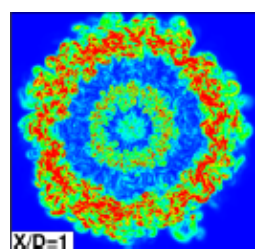

(c) $\mathrm{JW}-\mathrm{T} 5-\mathrm{H} / 10$

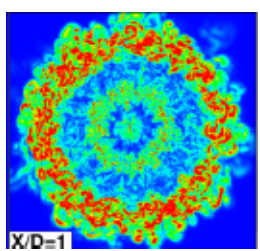

(d) $\mathrm{Jw}-\mathrm{T} 10-\mathrm{H} / 10$

Fig. 12 Vorticity magnitude in cross plane $x / D=1$
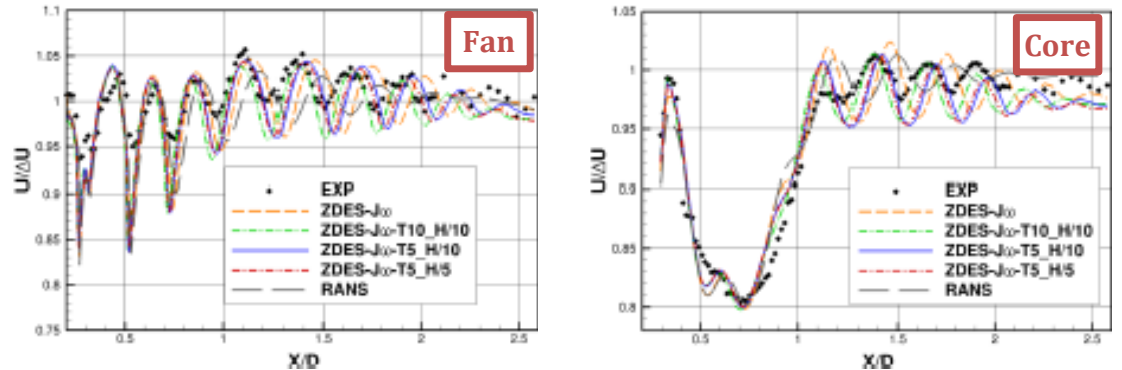

Fig. 13 Time-averaged streamwise velocity in the fan and core streams (red lines in Fig. 7)

\section{Summary and conclusion}

In both cases investigated in this paper, the introduction of upstream turbulence at the nozzle inlet in the jets core has led to improved agreement with the experimental data with regards to:

(1) The jet flow development, in terms of potential core length and shock-cell location, thanks to a better reproduction in the simulations of the experimental aerodynamic conditions;

(2) The RANS-to-LES transition in the shear layers, which is partly attributed to the penetration of the injected fluctuations inside the nozzle boundary layers which promotes the development of LES content in the shear layer. Of interest, it was not observed that this behavior impaired the shielding of the attached boundary layers treated in RANS mode with the ZDES mode 2 (which provides a RANS/LES switch without any user intervention).

Therefore these results promote a systematic introduction of upstream turbulence in eddy-resolving simulations of jets, and emphasize the need for more detailed experimental data on this matter. However, only aerodynamic data has been analyzed so far. Future work will be devoted to the assessment of the jet noise modification due to the upstream turbulence rate.

Acknowledgments The simulations presented in section 3 were performed using HPC resources from GENCI-TGCC (Grant 2014-t20142a7215). 


\section{References}

[1] S. Deck, "Recent Improvements of the Zonal Detached Eddy Simulation (ZDES) Formulation," Theoretical and Computational Fluid Dynamics, vol. 26, pp. 523-550, 2012. doi: 10.1007/s00162-011-0240-z.

[2] S. Deck, F. Gand, V. Brunet and S. Ben Khelil, "High-fidelity simulations of unsteady civil aircraft aerodynamics: stakes and perspectives. Application of zonal detached eddy simulation," Philosophical Transactions of the Royal Society A, 2014. doi: 10.1098/rsta.2013.0325.

[3] M. Shur, P. Spalart, M. Strelets and A. Travin, "An Enhanced Version of DES with Rapid Transition from RANS to LES in Separated Flows," Flow, Turbulence and Combustion, 2015. doi: 10.1007/s10494-015-9618-0.

[4] V. Brunet, "Random Flow Generation Technique for Civil Aircraft Jet Simulations with the ZDES Approach," in Notes on Numerical Fluid Mechanics and Multidisciplinary Design, vol. 117, Springer Berlin Heidelberg, 2012. doi: 10.1007/978-3-642-318184_17, pp. 193-204.

[5] F. Gand, "Investigation of turbulence development in incompressible jets with zonal detached eddy simulation (ZDES) and synthetic turbulent inflow," International Journal of Heat and Fluid Flow, pp. --, 2016. doi: 10.1016/j.ijheatfluidflow.2016.06.003.

[6] J. C. Tyacke, M. Mahak and P. G. Tucker, "Large-Scale Multifidelity, Multiphysics, Hybrid Reynolds-Averaged Navier-Stokes/Large-Eddy Simulation of an Installed Aeroengine," Journal of Propulsion and Power, 2016. doi: 10.2514/1.B35947.

[7] P. Spalart, W. Jou, M. Strelets and S. Allmaras, "Comments on the feasibility of LES for wings, and on a hybrid RANS/LES approach," in First AFOSR International Conference on DNS/LES., 1997.

[8] P. Spalart, S. Deck, M. Shur, K. Squires, M. Strelets and A. Travin, "A New Version of Detached-eddy Simulation, Resistant to Ambiguous Grid Densities," Theoretical and Computational Fluid Dynamics, vol. 20, pp. 181-195, 2006. doi: 10.1007/s00162-0060015-0.

[9] S. Davoust, L. Jacquin and B. Leclaire, "Dynamics of $m=0$ and $m=1$ Modes and of Streamwise Vortices in a Turbulent Axisymmetric Mixing Layer," Journal of Fluids Mechanics, vol. 709, pp. 408-444, 2012. doi: 10.1017/jfm.2012.342.

[10] L. Cambier, S. Heib and S. Plot, "The Onera elsA CFD software: input from research and feedback from industry," Mechanics and Industry, vol. 14, no. 3, pp. 159-174, 2013. doi: 10.1051/meca/2013056.

[11] I. Mary and P. Sagaut, "Large Eddy Simulation of Flow around an Airfoil Near Stall," AIAA Journal, vol. 40, no. 6, pp. 1139-1145, 2002.

[12] N. Jarrin, R. Prosser, J.-C. Uribe, S. Benhamadouche and D. Laurence, "Reconstruction of Turbulent Fluctuations for Hybrid RANS/LES Simulations using a Synthetic-Eddy Method," International Journal of Heat and Fluid Flow, vol. 30, no. 3, pp. 435-442, 2009. doi: 10.1016/j.ijheatfluidflow.2009.02.016.

[13] J. Verrière, F. Gand and S. Deck, "Zonal Detached-Eddy Simulations of a Dual-Stream Jet," AIAA Journal, pp. 1-15, 2016. doi: 10.2514/1.J054896.

[14] A. Smirnov, S. Shi and I. Celik, "Random Flow Generation Technique for Large Eddy Simulations and Particle-Dynamics Modeling," Journal of Fluids Engineering, vol. 123, no. 2, pp. 359-371, 2001. doi: 10.1115/1.1369598.

[15] J. Marty, B. Michel, N. Lantos and V. Bonneau, "LES and hybrid RANS/LES simulations of turbomachinery flows using high order methods," in Proceedings of ASME Turbo Expo 2015: Turbine Technical Conference and Exposition, GT2015, June 15 - 19, 2015, Montréal, Canada. Paper GF2015-42134, 2015. 